\title{
A 2D Metal Compound Shows a Superconducting Surprise
}

\author{
A cesium-rich "kagome" metal is both a topological insulator and a \\ superconductor, making it a compelling material for future quantum \\ technologies.
}

By Rachel Berkowitz

$\Gamma$ or quantum computing applications, a topological superconductor is the stuff of dreams. Such a material could protect qubits from the environment, leading to an extremely long coherence time. Researchers think that a new class of materials called kagome metals might be topological superconductors, as they have topological electronic structures, and they can host superconducting correlated-electron phenomena. But researchers have struggled to simultaneously realize both properties in these metals. Now Brenden Ortiz of the University of California, Santa Barbara, and colleagues have synthesized a superconducting kagome metal that has topologically protected surface states [1]. Their material provides a platform for exploring how superconductivity emerges from electronic structures in topological materials.

Ortiz and his colleagues synthesized a cesium-rich material that forms a layered crystalline metal. The lattice structure of each layer resembles that of a Japanese basket-weaving pattern
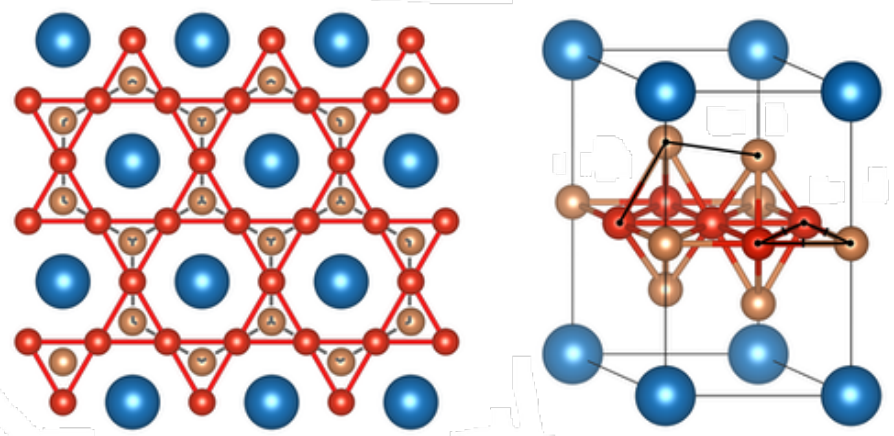

known as kagome. Taking a 2D thin film of this material, they characterized its electronic structure using spectroscopy measurements and calculations, revealing that it supports edge or surface states that act as one-way conductance channels. They also probed the metal's magnetization, heat capacity, and electrical resistivity, showing that it starts superconducting at $2.5 \mathrm{~K}$. That's surprising, the researchers say, because until now, only 3D versions of kagome metals have demonstrated superconductivity.

The researchers note that the superconducting temperature of this cesium-rich metal does not make it a sought-after high-temperature superconductor. The superconductivity also has no obvious connection to any topological feature. Despite those limitations, they still think that the metal presents a model material for probing how superconducting electrons and surface-state electrons influence each other's behavior.

Rachel Berkowitz is a Corresponding Editor for Physics based in Vancouver, Canada.

\section{REFERENCES}

1. B. Ortiz et al., " $\mathrm{CsV}_{3} \mathrm{Sb}_{5}: \mathrm{A} \mathbb{Z}_{2}$ topological kagome metal with a superconducting ground state," Phys. Rev. Lett. 125, 247002 (2020).

Credit: B. Ortiz et al. [1] 